\title{
THE SPHERICAL DERIVATIVE AND NORMAL FUNCTIONS
}

\author{
PETER LAPPAN
}

1. Let $D$ denote the unit disc in the complex plane. A function $f$ meromorphic in $D$ is called a normal function if there exists a constant $c_{1}(f)$ such that

for each $z \in D$, where

$$
\left(1-|z|^{2}\right) f^{\#}(z) \leqq c_{1}(f)
$$

$$
f^{\#}(z)=\frac{\left|f^{\prime}(z)\right|}{1+|f(z)|^{2}}
$$

is called the spherical derivative of $f$. Let $W$ denote the Riemann sphere and let $\chi(a, b)$ denote the chordal distance between the complex values $a$ and $b$, considered as points on the sphere, that is

$$
\chi(a, b)= \begin{cases}0 & \text { if } a=b, \\ \frac{|a-b|}{\sqrt{1+|a|^{2}} \sqrt{1+|b|^{2}}} & \text { if } a \neq \infty \neq b, \\ \frac{1}{\sqrt{1+|a|^{2}}} & \text { if } a \neq \infty=b .\end{cases}
$$

Thus

$$
f^{\#}(z)=\lim _{t \rightarrow z} \frac{\chi(f(t), f(z))}{|t-z|}
$$

and if $z_{1}$ and $z_{2}$ are two points in $D$ and $L$ is the line segment between them we have that

$$
\chi\left(f\left(z_{1}\right), f\left(z_{2}\right)\right) \leqq \int_{L} f^{\#}(z)|d z| .
$$

It is easy to show that $f$ is a normal meromorphic function if and only if the family $\left\{f(z ; \theta, a)=f\left(e^{i \theta}(z+a) /(1+\bar{a} z)\right): 0 \leqq \theta<2 \pi, a \in D\right\}$ is a normal family in the sense of Montel (see for example [2]). If $z_{1}$ and $z_{2}$ are two points in $D$, let

$$
\varrho\left(z_{1}, z_{2}\right)=\frac{1}{2} \ln \frac{1+h\left(z_{1}, z_{2}\right)}{1-h\left(z_{1}, z_{2}\right)},
$$


where

$$
h\left(z_{1}, z_{2}\right)=\frac{\left|z_{1}-z_{2}\right|}{\left|1-\bar{z}_{1} z_{2}\right|}
$$

$\varrho\left(z_{1}, z_{2}\right)$ is called the hyperbolic distance between $z_{1}$ and $z_{2}$. Another characterization of normal meromorphic functions is that normal meromorphic functions are precisely those which are uniformly continuous when considered as functions from the metric space $D$ using the hyperbolic metric to the metric space $W$ using the chordal metric.

Recently, S. Yamashita proved the following result [4, Theorem 1, p. 139].

Theorem Y. If $f$ is a normal meromorphic function in $D$, then there exists a constant $c_{2}(f)$ such that

for each $z \in D$.

$$
\left(1-|z|^{2}\right)^{2} f^{\#}(z)\left(f^{\prime}\right)^{\#}(z) \leqq c_{2}(f)
$$

We use this theorem of Yamashita as our point of departure.

In Section 2, we generalize Theorem $\mathrm{Y}$ by proving that if $f$ is a normal meromorphic function in $D$, then for each positive integer $n \geqq 1$ there exists a constant $c_{n}(f)$ such that

$$
\left(1-|z|^{2}\right)^{n} \prod_{j=0}^{n-1}\left(f^{(j)}\right)^{\#}(z) \leqq c_{n}(f)
$$

for each $z \in D$. We also show by example that a natural form of a converse to this result is not valid.

In Section 3 we use similar methods to prove a result involving normal functions and expressions analogous to the spherical derivative.

We conclude the paper with some open questions.

We wish to express our thanks to Professor Douglas Campbell for suggesting to us the method of proof for Theorem 1 presented in Section 2. The author's original proof, which involves slightly different methods, is outlined in Section 4 in the remark following Question 1.

2. Our results depend upon two basic lemmas.

Lemma 1. Let $K$ be a positive real number and let $f$ be a normal meromorphic function in $D$. For each positive integer $n$ there exists a constant $E_{n}(f, K)$ such that

$$
\left(1-|z|^{2}\right)^{n}\left|f^{(n)}(z)\right| \leqq E_{n}(f, K)
$$

for each $z \in D$ such that $|f(z)| \leqq K$.

Proof. Since $f$ is a normal meromorphic function, we have that

$$
f^{\#}(z) \leqq c_{1}(f) /\left(1-|z|^{2}\right) \leqq c_{1}(f) /(|1-| z \mid) .
$$


Let $\sigma=\chi(K, 2 K)$, and let

$$
A=\min \left(\frac{1}{2}, \frac{\sigma}{2 c_{1}(f)}\right)
$$

Thus, if $z_{0} \in D$ such that $\left|f\left(z_{0}\right)\right| \leqq K$, we have that $\left|z-z_{0}\right| \leqq A\left(1-\left|z_{0}\right|\right)$ implies

$$
\chi\left(f(z), f\left(z_{0}\right)\right) \leqq \int_{L} f^{\#}(z)|d z| \leqq \sigma,
$$

where $L$ is the line segment between $z$ and $z_{0}$. It follows that $\left|z-z_{0}\right|<$ $<A\left(1-\left|z_{0}\right|\right)$ implies that $|f(z)| \leqq 2 K$. By Cauchy's inequality for derivatives, we obtain

$$
\left|f^{(n)}\left(z_{0}\right)\right| \leqq \frac{2 K n !}{A^{n}\left(1-\left|z_{0}\right|\right)^{n}}
$$

for each positive integer $n$. Thus, letting

we get

$$
E_{n}(f, K)=2 K n !(2 / A)^{n}
$$

$$
\left(1-|z|^{2}\right)^{n}\left|f^{(n)}(z)\right| \leqq 2^{n}(1-|z|)^{n}\left|f^{(n)}(z)\right| \leqq E_{n}(f, K),
$$

where $|f(z)| \leqq K$, and this is the desired inequality.

Remark. The result of Lemma 1 actually characterizes normal meromorphic functions. For if $f$ is a meromorphic function in $D$ which is not normal, then by [3, Theorem 1, p. 492] for each fixed $K>0$ and for each value $w$ such that $|w|<K$, we have

$$
\sup \left\{\left(1-|z|^{2}\right) f^{\#}(z): f(z)=w\right\}=\infty
$$

with at most four exceptions $w$.

Lemma 2. For each positive integer $n \geqq 2$ and each meromorphic function $f$ in $D$, we have

for each $z \in D$.

$$
\prod_{j=0}^{n-1}\left(f^{(j)}\right)^{\#}(z) \leqq \frac{\left|f^{(n)}(z)\right|}{1+|f(z)|^{2}} \prod_{j=1}^{n-1} \frac{1}{\max \left\{2, \mid \overline{\left.f^{(j)}(z) \mid\right\}}\right.}
$$

Proof. For each $z \in D$,

$$
\prod_{j=0}^{n-1}\left(f^{(j)}\right)^{\#}(z)=\prod_{j=0}^{n-1} \frac{\left|f^{(j+1)}(z)\right|}{1+\left|f^{(j)}(z)\right|^{2}}=\frac{\left|f^{(n)}(z)\right|}{1+|f(z)|^{2}} \prod_{j=1}^{n-1} \frac{\left|f^{(j)}(z)\right|}{1+\left|f^{(j)}(z)\right|^{2}} .
$$

We remark that for each real number $x$ it is true that

$$
\frac{|x|}{1+x^{2}} \leqq \min \left\{\frac{1}{|x|}, \frac{1}{2}\right\}=\frac{1}{\max \{2,|x|\}}
$$


Thus

$$
\prod_{j=0}^{n-1}\left(f^{(j)}\right)^{\#}(z) \leqq \frac{\left|f^{(n)}(z)\right|}{1+|f(z)|^{2}} \prod_{j=1}^{n-1} \frac{1}{\max \left\{2,\left|f^{(j)}(z)\right|\right\}},
$$

which is the desired inequality.

Theorem 1. Let $f$ be a normal meromorphic function in $D$. Then for each positive integer $n$ there exists a constant $c_{n}(f)$ such that

for each $z \in D$.

$$
\left(1-|z|^{2}\right)^{n} \prod_{j=0}^{n-1}\left(f^{(j)}\right)^{\#}(z) \leqq c_{n}(f)
$$

Proof. Letting $K=1$ in Lemma 1, then by Lemma 2 we have

$$
\left(1-|z|^{2}\right)^{n} \prod_{j=0}^{n-1}\left(f^{(j)}\right)^{\#}(z) \leqq\left(1-|z|^{2}\right)^{n}\left|f^{(n)}(z)\right| \leqq E_{n}(f, 1),
$$

where $z \in D$ and $|f(z)| \leqq 1$. Hence we need only consider the situation where $|f(z)|>1$.

Let $g(z)=1 / f(z)$. Then $g$ is a normal meromorphic function, since $f^{\#}(z)=$ $=g^{\#}(z)$, and $|g(z)|<1$ whenever $|f(z)|>1$. If we differentiate the equation $f(z) g(z) \equiv 1 n$-times, we have

and thus,

$$
\sum_{k=0}^{n}\left(\begin{array}{l}
n \\
k
\end{array}\right) f^{(k)}(z) g^{(n-k)}(z) \equiv 0
$$

$$
f^{(n)}(z) g(z)=-\sum_{k=0}^{n-1}\left(\begin{array}{l}
n \\
k
\end{array}\right) f^{(k)}(z) g^{(n-k)}(z)
$$

Noting that $g(z)=1 / f(z)$, this means

$$
\left|f^{(n)}(z)\right| \leqq \sum_{k=0}^{n-1}\left(\begin{array}{l}
n \\
k
\end{array}\right)\left|f(z) f^{(k)}(z) g^{(n-k)}(z)\right| .
$$

Multiplying both sides of this inequality by the factor

$$
\frac{\left(1-|z|^{2}\right)^{n}}{\left(1+|f(z)|^{2}\right) \prod_{j=1}^{n-1} \max \left\{2,\left|f^{(j)}(z)\right|\right\}}
$$

we get, after combining similar factors of numerator and denominator on the right and using Lemma 1,

$$
\begin{aligned}
\frac{\left|f^{(n)}(z)\right|\left(1-|z|^{2}\right)^{n}}{\left(1+|f(z)|^{2}\right) \prod_{j=1}^{n-1} \max \left\{2,\left|f^{(j)}(z)\right|\right\}} & \leqq \sum_{k=0}^{n-1}\left(\begin{array}{l}
n \\
k
\end{array}\right)\left|g^{(n-k)}(z)\right|\left(1-|z|^{2}\right)^{n-k} \\
& \leqq \sum_{k=0}^{n-1}\left(\begin{array}{l}
n \\
k
\end{array}\right) E_{n-k}(g, 1)
\end{aligned}
$$


whenever $\mid f(z)>1$. Hence, if

$$
c_{n}(f)=\max \left\{E_{n}(f, 1), \sum_{k=0}^{n-1}\left(\begin{array}{l}
n \\
k
\end{array}\right) E_{n-k}(g, 1)\right\}
$$

we have, from Lemmas 1 and 2 ,

$$
\left(1-|z|^{2}\right)^{n} \prod_{j=0}^{n-1}\left(f^{(j)}\right)^{\#}(z) \leqq c_{n}(f)
$$

for each $z \in D$, and this completes the proof of the theorem.

We remark that Lemma 1 and Theorem 1 are essentially results about normal families. In particular, if we suppress the factors of $\left(1-|z|^{2}\right)$ and if we let $f$ vary within a normal family of meromorphic functions, it is easy to modify our proof to obtain the following result.

Theorem 2. Let $\mathfrak{F}$ be a normal family of meromorphic functions in D. For each positive integer $n$ and each compact subset $K$ of $D$ there exists a constant $L_{n}(K, \mathfrak{F})$ such that

$$
\prod_{j=0}^{n-1}\left(f^{(j)}\right)^{\#}(z) \leqq L_{n}(K, \mathfrak{F})
$$

for each $z \in K$ and each $f \in \mathfrak{r}$.

It is natural to consider whether any reasonable form of the converse to Theorem 1 is valid. Obviously, the converse of Theorem 1 is valid in its most natural form, since the words "for each positive integer $n$ " include the case $n=1$. However, we cannot replace the words "for each positive integer $n$ " by the words "for some positive integer $n$ " and hope to have a valid converse to Theorem 1 . The following theorem gives the appropriate counter-example to such an attempt at a converse.

Theorem 3. There exists a meromorphic function $f$ in $D$ such that $f$ is not a normal function, but

$$
\sup \left\{f^{\#}(z)\left(f^{\prime}\right)^{\#}(z)\left(1-|z|^{2}\right)^{2}: z \in D\right\}<\infty .
$$

Proof. Let $f(z)=(1-z) \exp \{1 /(1-z)\}$. If we use the auxiliary mapping $z(w)=$ $=1-(1 / w)$ we have that

$$
f(z)=f(z(w))=(1 / w) e^{w}
$$

where $\operatorname{Re} w>1 / 2$. From this we conclude that $f(z(w)) \rightarrow \infty$ as $w \rightarrow \infty$ along the real axis, while $f(z(w)) \rightarrow 0$ as $w \rightarrow \infty$ along the line $\operatorname{Re} w=1$. Hence, in $D, f(z)$ has the asymptotic value 0 at $z=1$, but $f(z) \rightarrow \infty$ as $z \rightarrow 1$ radially. If $f$ were a normal function, then by a result of Lehto and Virtanen [2, Theorem 2, p. 51], $f$ would have 0 as an angular limit at $z=1$, violating the fact of the radial limit 
$\infty$. Thus $f$ is not a normal function. However, we have both

and

$$
f^{\prime}(z)=\frac{z}{1-z} \exp \left\{\frac{1}{1-z}\right\}
$$

and hence

$$
f^{\prime \prime}(z)=\frac{1}{(1-z)^{3}} \exp \left\{\frac{1}{1-z}\right\}
$$

$$
\begin{aligned}
f^{\#}(z)\left(f^{\prime}\right)^{\#}(z)\left(1-|z|^{2}\right)^{2} & =\frac{\left|z /(1-z)^{4}\right||\exp \{1 /(1-z)\}|^{2}\left(1-|z|^{2}\right)^{2}}{\left(1+|(1-z) \exp \{1 /(1-z)\}|^{2}\right)\left(1+|z /(1-z) \exp \{1 /(1-z)\}|^{2}\right)} \\
& \leqq \frac{4}{|z|} \frac{|z /(1-z) \exp \{1 /(1-z)\}|^{2}}{1+|z /(1-z) \exp \{1 /(1-z)\}|^{2}} \leqq \frac{4}{|z|}
\end{aligned}
$$

for each $z \in D$. But both $f^{\#}(z)$ and $\left(f^{\prime}\right)^{\#}(z)$ are continuous finite valued functions in $D$, and hence, for $z \in D$,

where

$$
f^{\#}(z)\left(f^{\prime}\right)^{\#}(z)\left(1-|z|^{2}\right)^{2} \leqq \max \{B, 8\}
$$

$$
B=\sup \left\{f^{\#}(z)\left(f^{\prime}\right)^{\#}(z):|z| \leqq 1 / 2\right\} .
$$

It is of interest to note that the function $f(z)=(1-z) \exp \{1 /(1-z)\}$ is not a normal function, but all of its derivatives are normal functions, and the conclusion of Theorem 1 is satisfied by $f$ for $n \geqq 2$ (but not $n=1$ ).

The following example illustrates the power of Theorem 1 .

For each positive integer $n$, let $z_{n}=1-e^{-n}$ and let $x_{n}=1-(1 / 2) e^{-n}$. Then we have that $z_{n}<x_{n}<z_{n+1}$ such that $\varrho\left(z_{n}, z_{n+1}\right) \leqq 1$ for each positive integer $n$. Let

$$
B(z)=\prod_{n=1}^{\infty} \frac{z_{n}-z}{1-z_{n} z} .
$$

By a calculation due to Bagemihl and Seidel [1, Example 4, p. 11] there exists a positive number $\delta$ such that $\left|B\left(x_{n}\right)\right|>\delta$ for each $n$. Since the function $B(z)$ is a real valued function on the positive real axis, we can use the Mean Value Theorem to conclude both that $B^{\prime}(z)$ has a zero between $z_{n}$ and $z_{n+1}$ and that $\left|B^{\prime}(z)\right|$ assumes a value greater than $\lambda_{n}=2 \delta /\left(x_{n+1}-x_{n}\right)$ at some point in the interval between $x_{n}$ and $x_{n+1}$. Hence the function $\left|B^{\prime}(z)\right|$ assumes both the value 0 and the value $\lambda_{n}$ on the interval between $z_{n}$ and $z_{n+2}$. But $\varrho\left(z_{n}, z_{n+2}\right) \leqq 2$, and this behavior of $B^{\prime}(z)$ could not occur if $B^{\prime}(z)$ were a normal function (see, for example $\left[1\right.$, p. 5]). Thus $B^{\prime}(z)$ is not a normal function.

This argument can be repeated indefinitely to show that $B^{\prime \prime}(z), B^{\prime \prime \prime}(z)$ and all higher derivatives of $B(z)$ are not normal functions. But $B(z)$ is a bounded analytic function, and thus is a normal function. Hence, by Theorem 1, we have that

$$
\prod_{j=0}^{n-1}\left\{\left(B^{(j)}\right)^{\#}(z)\left(1-|z|^{2}\right)\right\} \leqq c_{n}(B)
$$


for each $z \in D$ and each positive integer $n$, even though each factor on the left, except for the factor corresponding to $j=0$, is unbounded in $D$.

3. For a meromorphic function $f$ in $D$ and a positive integer $n$ the expression

$$
\frac{\left|f^{(n)}(z)\right|}{1+|f(z)|^{n+1}}
$$

represents an extension of the spherical derivative of $f$. We will prove that this expression is meaningful when related to normal functions.

Theorem 4. If $f$ is a normal meromorphic function in $D$, then for each positive integer $n$ there exists a constant $P_{n}(f)$ such that

for each $z \in D$.

$$
\frac{\left|f^{(n)}(z)\right|\left(1-|z|^{2}\right)^{n}}{1+|f(z)|^{n+1}} \leqq P_{n}(f)
$$

Proof. The theorem is true for $n=1$, since this is our definition of a normal function. We now proceed by induction.

Suppose the theorem is true for each $k<n$. By Lemma 1, we have that

$$
\frac{\left|f^{(n)}(z)\right|\left(1-|z|^{2}\right)^{n}}{1+\mid f(z)^{\mid n+1}} \leqq\left|f^{(n)}(z)\right|\left(1-|z|^{2}\right)^{n} \leqq E_{n}(f, 1)
$$

where $|f(z)| \leqq 1$. Let $g(z)=1 / f(z)$. Then $|g(z)|<1$ whenever $|f(z)|>1$, and $g$ is a normal meromorphic function. If $|f(z)|>1$, we can differentiate the equation $f(z) g(z) \equiv 1 \quad n$-times to get, as before,

$$
\left|f^{(n)}(z)\right| \leqq \sum_{k=0}^{n-1}\left(\begin{array}{l}
n \\
k
\end{array}\right)\left|f(z) f^{(k)}(z) g^{(n-k)}(z)\right|
$$

and so, letting $n \geqq 1$ and $P_{0}(f)=1$, we get

$$
\begin{gathered}
\frac{\left|f^{(n)}(z)\right|\left(1-|z|^{2}\right)^{n}}{1+|f(z)|^{n+1}} \\
\leqq \sum_{k=0}^{n-1}\left\{\left(\begin{array}{l}
n \\
k
\end{array}\right) \frac{|f(z)|\left|f^{(k)}(z)\right|\left(1-|z|^{2}\right)^{k}}{1+|f(z)|^{k+1}} \cdot\left|g^{(n-k)}(z)\right|\left(1-|z|^{2}\right)^{n-k} \cdot\left(\frac{1+|f(z)|^{k+1}}{1+|f(z)|^{n+1}}\right)\right\} \\
\leqq \sum_{k=0}^{n-1}\left(\begin{array}{l}
n \\
k
\end{array}\right) P_{k}(f) E_{n-k}(g, 1)\left(\frac{|f(z)|+|f(z)|^{k+2}}{1+|f(z)|^{n+1}}\right) .
\end{gathered}
$$

But it is easy to see that $\left(x+x^{n+1}\right) /\left(1+x^{n+1}\right) \leqq 2$ whenever $x>1$, so that in our case,

$$
\frac{|f(z)|+|f(z)|^{k+2}}{1+|f(z)|^{n+1}} \leqq 2
$$


whenever $0 \leqq k \leqq n-1$ and $\mid f(z)>1$. Hence, we have

$$
\frac{\left|f^{(n)}(z)\right|\left(1-|z|^{2}\right)^{n}}{1+|f(z)|^{n+1}} \leqq \sum_{k=0}^{n-1} 2\left(\begin{array}{l}
n \\
k
\end{array}\right) P_{k} E_{n-k}(g, 1)
$$

whenever $|f(z)|>1$. Hence, setting

$$
P_{n}(f)=\max \left\{E_{n}(f, 1), \sum_{k=0}^{n-1} 2\left(\begin{array}{l}
n \\
k
\end{array}\right) P_{k}(f) E_{n-k}(g ; 1)\right\},
$$

we obtain the desired result.

We remark that our proof can be modified to yield a theorem concerning a normal family, and we state the result without proof.

Theorem 5. Let $\mathfrak{F}$ be a normal family of meromorphic functions on $D$ and let $K$ be a compact subset of $D$. For each positive integer $n$ there exists a constant $P_{n}(K, \mathbb{J})$ such that

$$
\frac{\left|f^{(n)}(z)\right|\left(1-|z|^{2}\right)^{n}}{1+|f(z)|^{n+1}} \leqq P_{n}(K, \mathfrak{F})
$$

for each $z \in K$ and each $f \in \mathfrak{F}$.

4. We conclude with some open questions.

Question 1. Does there exist a meromorphic function $f$ normal in $D$ such that the sequence $\left\{c_{n}(f)\right\}$ is unbounded, where $\left\{c_{n}(f)\right\}$ is the sequence of constants in Theorem 1?

We remark that our proof does not seem to shed useful light on this question. The constants $E_{n}(f)$ can certainly become unbounded, as is evidenced by the function $f(z)=1 /(1-z)$ (using the point $z=0$ as a point of easy calculation), but the estimates made in the proof of Theorem 1 do not make use of all the factors appearing in the denominator. It is thus conceivable that the sequence $\left\{c_{n}(f)\right\}$ could be bounded for each fixed normal meromorphic function $f$.

We remark that an alternative proof of Theorem 1 is available, which might be of interest in connection with Question 1, and we will outline the argument here.

As noted before, we need only consider the situation when $|f(z)|>1$. Let $n>1$, and assume that

$$
\frac{\left|f^{(p)}(z)\right|\left(1-|z|^{2}\right)^{p}}{\left(1+|f(z)|^{2}\right) \prod_{q=1}^{p-1} \max \left\{2,\left|f^{(q)}(z)\right|\right\}} \leqq c_{p}(f)
$$

for each $p<n$. We note that this inequality is true for $p=1$, since $f$ is a normal function. Now let $g(z)=1 / f(z)$. Then $g^{\prime}(z)=-f^{\prime}(z) /(f(z))^{2}$, and thus $f^{\prime}(z)=$ $=-(f(z))^{2} g^{\prime}(z)$ : Differentiating this equation $(n-1)$-times, and using the triangle inequality, we get

$$
\left|f^{(n)}(z)\right| \leqq \sum a_{i j k}\left|f^{(i)}(z) f^{(j)}(z) g^{(k)}(z)\right|,
$$


where the summation is taken over all non-negative integers, $i, j, k$ satisfying $i+j+k=n$ and $k \geqq 1$, and the constants $a_{i j k}$ are independent of $f$. Multiplying both sides of this inequality by

$$
\frac{\left(1-|z|^{2}\right)^{n}}{\left(1+|f(z)|^{2}\right) \prod_{q=1}^{n-1} \max \left\{2,\left|f^{(q)}(z)\right|\right\}}
$$

we can consider separately those terms on the right where $i \neq j$, and those terms where $i=j$. If $i \neq j$, the factors $f^{(i)}(z)$ and $f^{(j)}(z)$ are dominated by like factors in the denominator. If $i=j=0$, the term $\left|f^{(0)}(z)\right|^{2}=|f(z)|^{2}$ is dominated by the factor $\left(1+|f(z)|^{2}\right)$ in the denominator. If $i=j \neq 0$, then one of the factors $\left|f^{(i)}(z)\right|$ is dominated by a like factor in the denominator and we are left with a term of the form

$$
a_{i j k}\left[\frac{\left|f^{(i)}(z)\right|\left(1-|z|^{2}\right)^{i}}{\left(1+|f(z)|^{2}\right) \prod_{j=1}^{i-1} \max \left\{2,\left|f^{(j)}(z)\right|\right\}}\right] \frac{\left|g^{(k)}(z)\right|\left(1-|z|^{2}\right)^{n-i}}{\prod_{p=i+1}^{n-1} \max \left\{2,\left|f^{(p)}(z)\right|\right\}},
$$

where the inductive hypothesis gives that the term in the brackets [ ] is less than $c_{i}(f)$. The rest of the argument now proceeds as in Section 2 , since we have a finite sum of terms each of which is bounded by constants already assumed (inductively) to exist.

Question 2. Suppose that for a given meromorphic function $f$ in $D$ there exists a specific positive integer $n_{0}$ and a constant $Q$ such that

$$
\left(1-|z|^{2}\right)^{n_{0}} \prod_{k=0}^{n_{0}-1}\left(f^{(k)}\right)^{\#}(z) \leqq Q
$$

for each $z \in D$. Must one of the functions $f, f^{1}, \ldots, f^{\left(n_{0}\right)}$ be a normal function?

Clearly, if $n_{0}=1$ then $f$ is a normal function. However, Theorem 3 gives an example of a function $f$ for which $n_{0}=2$ and $f$ is not a normal function but $f^{\prime}$ is a normal function.

Question 3. Is the converse of Theorem 4 true, that is, if there exist $n_{0}>1$ and a constant $P$ such that

$$
\frac{\left|f^{\left(n_{0}\right)}(z)\right|}{1+|f(z)|^{n_{0}+1}}\left(1-|z|^{2}\right)^{n_{0}} \leqq P
$$

for each $z \in D$, is $f$ a normal function?

We exclude the case $n_{0}=1$ from the question because the answer there is affirmative from our definition of a normal function.

We remark that for the function $f(z)=1 /(1-z)$ we have

$$
\frac{f^{(n)}(0)}{1+|f(0)|^{n+1}}=\frac{n !}{2}
$$

so that $\boldsymbol{P}_{n}(f) \rightarrow \infty$ in this case. Thus the analogue of Question 1 for the sequence $\left\{P_{n}(f)\right\}$ in Theorem 4 has an affirmative answer. 


\title{
References
}

[1] Bagemihl, F., and W. Seidel: Sequential and continuous limits of meromorphic functions. - Ann. Acad. Sci. Fenn. Ser. A I 280, 1960, 1-17.

[2] Lehto O., and K. I. VIRTANEN: Boundary behaviour and normal meromorphic functions. - Acta Math. 97, 1957, 47-65.

[3] LAPPAN, P.: A criterion for a meromorphic function to be normal. - Comment. Math. Helv. 49, 1974, 492-495.

[4] YAMASHITA, S.: On normal meromorphic functions. - Math. Z. 141, 1975, 139-145.

\author{
Michigan State University \\ Department of Mathematics \\ East Lansing, Michigan 48824 \\ USA
}

Received 16 September 1977 